\title{
Circular polarization signals of cloudy (exo)planets
}

\author{
L. Rossi and D. M. Stam
} Faculty of Aerospace Engineering, Delft University of Technology, Kluyverweg 1, 2629 HS Delft, The Netherlands
e-mail: d.m.stam@tudelft.nl, 1.c.g.rossi@tudelft.nl

Received 11 January 2018 / Accepted 2 May 2018

\begin{abstract}
Context. The circular polarization of light that planets reflect is often neglected because it is very small compared to the linear polarization. It could, however, provide information on a planet's atmosphere and surface, and on the presence of life, because homochiral molecules that are the building blocks of life on Earth are known to reflect circularly polarized light.

Aims. We compute $P_{\mathrm{c}}$, the degree of circular polarization, of light that is reflected by rocky (exo)planets to provide insight into the viability of circular spectropolarimetry for characterizing (exo)planetary atmospheres.

Methods. We compute the $P_{\mathrm{c}}$ of light that is reflected by rocky (exo)planets with liquid water or sulfuric acid solution clouds, both spatially resolved across the planetary disk and, for planets with patchy clouds, integrated across the planetary disk, for various planetary phase angles $\alpha$.

Results. The optical thickness and vertical distribution of the atmospheric gas and clouds, the size parameter and refractive index of the cloud particles, and $\alpha$ all influence $P_{\mathrm{c}}$. Spatially resolved, $P_{\mathrm{c}}$ varies between $\pm 0.20 \%$ (the sign indicates the polarization direction). Only for small gas optical thicknesses above the clouds do significant sign changes (related to cloud particle properties) across the planets' hemispheres occur. For patchy clouds, the disk-integrated $P_{\mathrm{c}}$ is typically smaller than $\pm 0.025 \%$, with maximum for $\alpha$ between $40^{\circ}$ and $70^{\circ}$, and $120^{\circ}$ to $140^{\circ}$. As expected, the disk-integrated $P_{\mathrm{c}}$ is virtually zero at $\alpha=0^{\circ}$ and $180^{\circ}$. The disk-integrated $P_{\mathrm{c}}$ is also very small at $\alpha \approx 100^{\circ}$.

Conclusions. Measuring circular polarization signals appears to be challenging with current technology. The small atmospheric circular polarization signal could, however, allow the detection of circular polarization due to homochiral molecules such as those associated with life on Earth. Confirmation of the detectability of such signals requires better knowledge of the strength of circular polarization signals of biological sources and in particular of the angular distribution of their scattering.
\end{abstract}

Key words. polarization - techniques: polarimetric - radiative transfer - planets and satellites: atmospheres

\section{Introduction}

Polarimetry has been shown to be a strong tool for the characterization of solar system planets, with the successful characterization of the clouds of Venus (Hansen \& Hovenier 1974), the studies of atmospheres of other planets such as those of Jupiter (see e.g. McLean et al. 2017, and references therein), Saturn (Tomasko \& Doose 1984), Uranus, and Neptune (Schmid et al. 2006), and airless bodies such as comets and asteroids (Kiselev et al. 2015; Cellino et al. 2015, and references therein). There is also a great potential for polarimetry of exoplanets because the light emitted by solar-type stars can be assumed to be unpolarized when integrated across their disk (Kemp et al. 1987), while it is usually polarized upon reflection by a planet. Polarimetry can thus increase the contrast between background starlight and a planet (Seager et al. 2000; Lucas et al. 2009), and ease the detection and confirmation of an exoplanet. And, because the degree and direction of polarization are sensitive to the composition and structure of a planetary atmosphere and surface, polarimetry could also help to characterize an exoplanet (see e.g. Seager et al. 2000; Stam et al. 2004; Stam 2008; Karalidi et al. 2012).

Like the state of linear polarization, the state (degree and direction) of circular polarization can contain information about the structure and composition of a planetary atmosphere and about the properties of a planetary surface. Circular polarization is typically induced through the multiple scattering of light by non-gaseous, atmospheric particles (Hansen \& Hovenier 1974), or through the multiple reflection of light by a rough surface (Kemp \& Wolstencroft 1971), but, interestingly, it also displays potential for use in the detection of life (MacDermott et al. 1996; Bailey 2000; Sparks et al. 2005). This is due to the preferred orientation of the chiral molecules that make up organisms on Earth, referred to as homochirality (Bonner 1991), which induces distinctive circular polarization variations with wavelengths in reflected and transmitted light (Sparks et al. 2009, 2012b; Nagdimunov et al. 2013; Patty et al. 2017), although measurements on two types of cyanobacteria species by Martin et al. (2016) also suggest that measured circular polarization signals can be due to internal reflections and thus not necessarily due to homochirality.

While many studies have concentrated on the state of the linear polarization of light, few have considered circular polarization of reflected sunlight. Indeed, only a handful of measurements of the state of circular polarization of solar system planets are available (Kemp \& Wolstencroft 1971; Swedlund et al. 1972; Wolstencroft 1976; Kawata 1978; Meierhenrich et al. 2002; Sparks et al. 2012b), all obtained with Earth-based telescopes. As far as we know, there has not been a space-based instrument with circular polarization measurement capabilities targeting planets. Measuring the circular polarization requires more complicated instrumentation (often with more, and moving, parts) than measuring the linear polarization (Sparks et al. 2012a), also because the degree of circular polarization is usually 
very small, especially when compared to the degree of linear polarization. The instrument requirements for circular polarization instruments, in particular the limits on the cross-talk, are thus very high. Recent advances in the design and production of optical elements, however, are increasing the viability of circular polarimetry as a tool for planetary characterization. In addition, the possibility of detecting circular polarization of light reflected by chiral molecules and thus possibly of detecting life, has increased the interest in circular polarization signals of (exo)planets.

Therefore, this study acts as a starting point for an investigation into the feasibility of the use of circular polarimetry for planetary observations and the identification of homochirality. Circular polarization can be induced by a planetary atmosphere, surface, or a biological presence and it is important to understand the influence that these processes have on the total circular polarization profile of the planet. However, the degree of circular polarization coming from the surface of the planet is irrelevant if it is not visible due to an overwhelming signal from the atmosphere. Therefore, it is vital that the influence of the atmospheric properties on the circular polarization signal of an exoplanet is first studied.

In this paper, we investigate the effects of clouds on the degree and direction of circular polarization of light reflected by Earth-like planets for both spatially resolved and disk-integrated measurements. The latter would be representative for (future) measurements of starlight reflected by Earth-like exoplanets. In Sect. 2, we describe the numerical algorithm to compute the polarization signals and provide the physical properties of the atmospheres, including the clouds, and the surfaces that cover our model planets. In Sect. 3, we present calculated polarization signals for planets with different cloud optical thicknesses, microphysical cloud properties, cloud top altitude, and fractional cloud coverage. In Sect. 4, we discuss the limits of our simulations and the possible issues that observers might face. Finally, in Sect. 5, we summarize our results.

\section{Numerical algorithms}

\subsection{Defining fluxes and polarization}

We describe the starlight that is incident on a planet and the starlight that is reflected by the planet by a Stokes vector $\mathbf{F}$, as (see e.g. Hansen \& Travis 1974; Hovenier et al. 2004)

$\mathbf{F}=\left[\begin{array}{c}F \\ Q \\ U \\ V\end{array}\right]$,

where $F$ describes the total flux, $Q$ and $U$ the linearly polarized fluxes, and $V$ the circularly polarized flux. We express these fluxes in $\mathrm{W} \mathrm{m}^{-2}$. Linearly polarized fluxes $Q$ and $U$ are defined with respect to a reference plane, for which we use the planetary scattering plane, that is, the plane that contains (the centre of) the star, the planet, and the observer. Parameters $Q$ and $U$ can straightforwardly be redefined with respect to another reference plane, such as the optical plane of an instrument, using a so-called rotation matrix (see Hovenier \& van der Mee 1983; for the definition).

The circularly polarized flux $V$ is "the excess of flux transmitted by an instrument that passes right-handed circular polarization, over that transmitted by an instrument that passes left-handed circular polarization" (Hansen \& Travis 1974).
Regarding starlight that is reflected by a planet and that arrives at an observer, parameter $V$ will thus be positive if the observer "sees" the electric vector rotating in the anticlockwise direction, and $V$ will be negative if the observer "sees" a rotation in the clockwise direction.

We assume that the starlight that is incident on a planet is unidirectional and unpolarized. The justification of the latter is based on the very small values of the disk-integrated polarization of solar-type stars. Kemp et al. (1987) gave measurements of the linear and circular polarization of the Sun: their diskintegrated measurements yield maximal values of $0.8 \times 10^{-6}$ for the linear polarization, and $-1 \times 10^{-6}$ for the circular polarization, with a $B$ filter. Locally, disturbances on the stellar disk such as stellar spots or transiting planets could produce a break in the symmetry of the stellar disk and generate non-zero diskintegrated values of polarization. The values expected from such disturbances are on the order of $10^{-6}$ for the degree of linear polarization (Berdyugina et al. 2011; Kostogryz et al. 2015). The incident light is thus described as $\mathbf{F}_{0}=F_{0} \mathbf{1}$, with $\pi F_{0}$ the stellar flux measured perpendicular to the direction of propagation, and 1 the unit column vector.

Starlight that is reflected by an orbiting planet will usually be polarized because it has been scattered by gases and aerosols or cloud particles in the planetary atmosphere and/or has been reflected by the surface. The degree of polarization of the reflected starlight is defined as

$P=\frac{\sqrt{Q^{2}+U^{2}+V^{2}}}{F}$

the degree of linear polarization as

$P_{1}=\frac{\sqrt{Q^{2}+U^{2}}}{F}$

and the degree of circular polarization as

$P_{\mathrm{c}}=\frac{V}{F}$.

Following the description above, if $P_{\mathrm{c}}>0$, the observer "sees" the electric vector of the light rotating in the anticlockwise direction, and if $P_{\mathrm{c}}<0$, the observer "sees" a rotation in the clockwise direction.

\subsection{The radiative transfer algorithm}

In this paper, we will present computed (polarized) fluxes and polarization for both spatially resolved and spatially unresolved planets. The latter, for which we integrate the spatially resolved signals across the planetary disks, apply to (future) observations of exoplanets, while the former apply to observations of solar system planets and also helps us to understand the computed exoplanet signals.

For our spatially resolved computations, we first divide the two-dimensional (2D) planetary disk facing the observer into equal-sized, square pixels, with 30 or 100 pixels along the equator of the disk, which is assumed to run horizontally through the middle of the disk. Then, we project the centre of each pixel onto the three-dimensional (3D) planet and determine the following angles for each projected pixel centre on the planet: $\theta_{0}$, the angle between the local vertical and the direction towards the illuminating sun or star, $\theta$, the angle between the local vertical and the direction towards the observer, and the azimuthal difference angle $\phi-\phi_{0}$, which is the angle between the plane containing 
the local vertical and the propagation direction of the incident light and the plane containing the local vertical and the direction towards the observer (for a detailed definition, see de Haan et al. 1987). The angles depend on the latitude and longitude of the projected pixel centre, and $\theta_{0}$ and $\phi_{0}$ also depend on the planet's phase angle $\alpha$, that is, the angle between the star and the observer as measured from the centre of the planet $\left(0^{\circ} \leq \alpha \leq 180^{\circ}\right)$.

Next, we calculate for each pixel, the Stokes vector (see Eq. (1)) of the starlight that is reflected at the projected pixel centre on the planet, using

$\mathbf{F}\left(\theta, \theta_{0}, \phi-\phi_{0}\right)=\cos \theta_{0} \mathbf{R}_{1}\left(\theta, \theta_{0}, \phi-\phi_{0}\right) \quad \mathbf{F}_{0}$,

with $\mathbf{R}_{1}$ the first column of the $4 \times 4$ local planetary reflection matrix. Only the first column is relevant, since the incoming starlight is assumed to be unpolarized. The actual area on the planet that is covered by a projected pixel varies with latitude and longitude on the planet, but because all pixels have the same size, their respective Stokes vectors as computed according to Eq. (5) contribute equally to the planetary signal. With our pixels covering the planetary disk, we can straightforwardly model horizontally inhomogeneous planets by choosing different atmosphere and/or surface models for different pixels. The physical properties of our models are described in Sect. 2.3.

Given an atmosphere-surface model for a given pixel, we use an adding-doubling algorithm that fully includes polarization for all orders of scattering (de Haan et al. 1987) to compute the local planetary reflection matrix (see Eq. (5)). Rather than embarking on a separate radiative transfer computation for every pixel, we choose to first compute and store the coefficients $\mathbf{R}_{1}^{m}\left(\theta, \theta_{0}\right)$ ( $0 \leq m<M$, with $M$ the total number of coefficients) of the expansion of $\mathbf{R}_{1}\left(\theta, \theta_{0}, \phi-\phi_{0}\right)$ into a Fourier series (see de Haan et al. 1987, for the description of this expansion for the reflection matrix) for the different atmosphere-surface models that occur on our model planet. Our adding-doubling algorithm computes these coefficients at values of $\cos \theta_{0}$ and $\cos \theta$ that coincide with Gaussian abscissae (the total number of which is user-defined), and additionally for $\theta_{0}=0^{\circ}$ and $\theta=0^{\circ}$. Given a pixel with its local angles $\theta_{0}, \theta$, and $\phi-\phi_{0}$, we can efficiently compute its $\mathbf{R}_{1}$ by summing up (see de Haan et al. 1987) the Fourier coefficients stored for the relevant atmosphere-surface model, interpolating for values of $\theta_{0}$ and $\theta$ when necessary.

Each pixel is independent from its neighbours; there is no 3D cross-pixel propagation of light. This remains a good approximation considering that the projected area of each pixel on the planet is quite large, meaning that the cross-pixel propagation will occur on scales quite small compared to the pixel size. Furthermore, the case most likely to be affected is that of an inhomogeneous cloud cover (in particular at the edge of clouds). But in our simulations of inhomogeneous covers (see Sect. 3.2), we consider averages over 300 cloud realisations, which we expect should make the effect of horizontal propagation of light negligible.

To compute the disk-integrated Stokes vector, we sum up the Stokes vectors pertaining to the pixels covering the planetary disk. A locally reflected Stokes vector as computed by our adding-doubling algorithm is defined with respect to the local meridian plane, which contains both the local vertical direction and the direction towards the observer. This is perfectly fine when our aim is to compare degrees of polarization between different pixels, because degrees of polarization are independent of the reference plane. However, for every pixel we redefine the local vector to the common reference plane

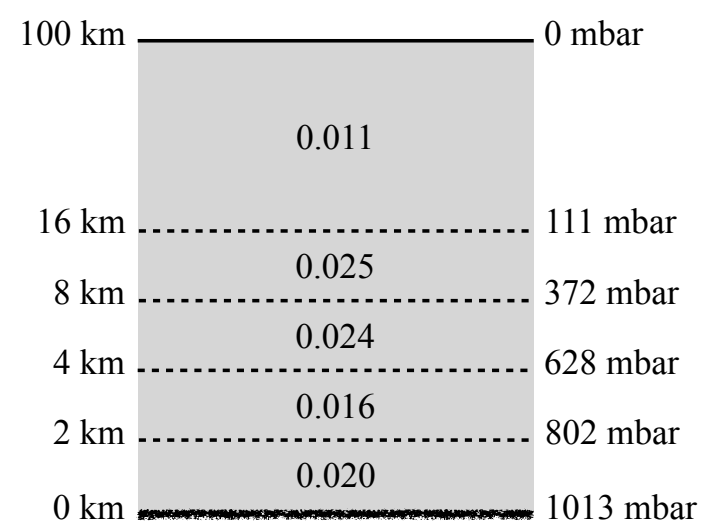

Fig. 1. Gaseous optical thickness $b_{\mathrm{m}}$ at $550 \mathrm{~nm}$ in layers comprising the model atmosphere. The altitude and pressure of the levels bounding the layers are also given. The total gaseous optical thickness of the model atmosphere is 0.096 .

(the planetary scattering plane) using the so-called rotation matrix $\mathbf{L}$ and $\beta$, the angle between the local meridian plane and the planetary scattering plane (see Hovenier \& van der Mee 1983). This is done before summing up local Stokes vectors for the disk-integration.

We normalize disk-integrated flux vectors such that the reflected flux at $\alpha=0^{\circ}$ equals the planet's geometric albedo (see Stam et al. 2004; Stam 2008). The degrees of total, linear, and circular polarization, $P, P_{1}$, and $P_{\mathrm{c}}$, are relative measures (see Eqs. (2)-(4)) and thus independent of a normalization. The degrees of polarization computed in this paper pertain only to the planet. The polarization of the system as a whole would be lower as the star would add mostly unpolarized flux. We ignore sources of noise such as zodiacal or interstellar dust, or instrumental polarization.

\subsection{The model atmospheres and surfaces}

The atmosphere and surface of our model planets are assumed to be locally horizontally homogeneous and flat. Unless stated otherwise, the surface below the atmosphere is Lambertian, that is, an isotropic and completely depolarizing surface. Its albedo is indicated by $A_{\mathrm{s}}$. In this paper, we use $A_{\mathrm{s}}=0.0$ for the whole planetary surface, unless specified otherwise.

The atmosphere can be vertically inhomogeneous: it is composed of a stack of homogeneous layers. Each layer contains gaseous molecules that are anisotropic Rayleigh scatterers with a depolarization factor of 0.0279 (see Hansen \& Travis 1974), and, optionally, cloud particles. The amount of gas and cloud particles in a layer is represented by the optical thicknesses $b_{\mathrm{m}}$ and $b_{\mathrm{c}}$, respectively. The total gaseous optical thickness of our model atmosphere (thus summed over all layers) is 0.096, which is representative for the Earth's atmosphere at a wavelength of about $550 \mathrm{~nm}$ (see Stam 2008; for a detailed description of how $b_{\mathrm{m}}$ is computed). We ignore absorption by atmospheric gases. Figure 1 shows how this optical thickness is divided over five atmospheric layers. The cloud particles are distributed homogeneously within one of these layers.

The single scattering matrix of most types of atmospheric scatterers is given by (see Hansen \& Travis 1974)

$\mathbf{P}(\Theta)=\left[\begin{array}{cccc}P_{11}(\Theta) & P_{21}(\Theta) & 0 & 0 \\ P_{21}(\Theta) & P_{22}(\Theta) & 0 & 0 \\ 0 & 0 & P_{33}(\Theta) & -P_{34}(\Theta) \\ 0 & 0 & P_{34}(\Theta) & P_{44}(\Theta)\end{array}\right]$, 
with $\Theta$ the single scattering angle $\left(0^{\circ} \leq \Theta \leq 180^{\circ}\right.$, with $\Theta=0^{\circ}$ indicating forward scattered light).

For (anisotropic) Rayleigh scattering, matrix element $P_{34}$ equals zero. Consequently, if Rayleigh scattering is the only scattering process in a planetary atmosphere, incident unpolarized light cannot become circularly polarized by scattering in the atmosphere, not even when it is scattered multiple times. For atmospheric particles that are large compared to the wavelength of the incident light, such as cloud particles, element $P_{34}$ is nonzero for most values of $\Theta$. Unpolarized incident light can thus become circularly polarized when it is scattered at least twice by such particles (or first at least once by a Rayleigh scatterer and then by a large aerosol or cloud particle).

We assume that the particles constituting the clouds are spherical and internally homogeneous. We compute their single scattering matrix with the Mie-scattering algorithm described by de Rooij \& van der Stap (1984). The sizes of the cloud particles follow the two-parameter gamma distribution, with an effective radius $r_{\text {eff }}$ and an effective variance $v_{\text {eff }}$. Our standard values for $r_{\text {eff }}$ and $v_{\text {eff }}$ are $8.0 \mu \mathrm{m}$ and 0.1, respectively, similar to Earth cloud values from Han et al. (1994). For the refractive index we use $1.33+10^{-8} i$, which is representative for liquid water at $550 \mathrm{~nm}$ (Hale \& Querry 1973). The single scattering albedo of these particles is close to one. We will also use Venus-like cloud particles, described by the same size distribution, except with $r_{\text {eff }}=1.05 \mu \mathrm{m}, v_{\text {eff }}=0.07$, and with a refractive index of $1.44+0.015 i$, which is representative for a sulfuric acid solution at $500 \mathrm{~nm}$ (Hansen \& Hovenier 1974). The single scattering albedo of the Venus-like particles is 0.75 .

Figure 2 shows the elements of the scattering matrix for the two types of cloud particles. As can be seen from Eqs. (3) and (6), the ratio $P_{21} / P_{11}$ describes the degree of linear polarization of incident unpolarized light that has been scattered only once. In Fig. 2, we have included a minus sign for this ratio to indicate the direction of polarization of the scattered light: if $-P_{21} / P_{11}>0$, the scattered light is polarized perpendicular to the plane that contains both the incoming and the scattered beams, while if $-P_{21} / P_{11}<0$, it is polarized parallel to this plane.

The curves in Fig. 2 clearly show the strong forward scattering peak in the phase functions (elements $P_{11}$ ) of both cloud particle types, and the enhanced scattering representative for the first and second order rainbows, around $\Theta=140^{\circ}$ and $125^{\circ}$, respectively, in the phase function of the water cloud particles. These rainbow features also show up in other matrix elements, in particular in $-P_{21} / P_{11}$.

\subsection{The cloud cover}

To investigate the sensitivity of the circularly polarized flux due to the cloud optical thickness, cloud particle properties (size and composition), and cloud top altitude, we will show fluxes and degrees of polarization calculated for planets that are completely covered by a cloud deck. The disk-integrated circularly polarized flux (and degree of circular polarization) of a horizontally homogeneous planet will be zero, because the circularly polarized flux of a pixel on the northern hemisphere will be cancelled by that of the corresponding pixel on the southern hemisphere, as that would have the same absolute value but the opposite sign (this can be seen in Fig. 3). Thus, to simulate the circularly polarized fluxes of spatially unresolved planets, such as exoplanets, we will use horizontally inhomogeneous model planets that are asymmetric with respect to the planetary scattering plane due to patchy clouds. We will limit ourselves to a single type of cloud per planet, thus, the clouds in all cloudy
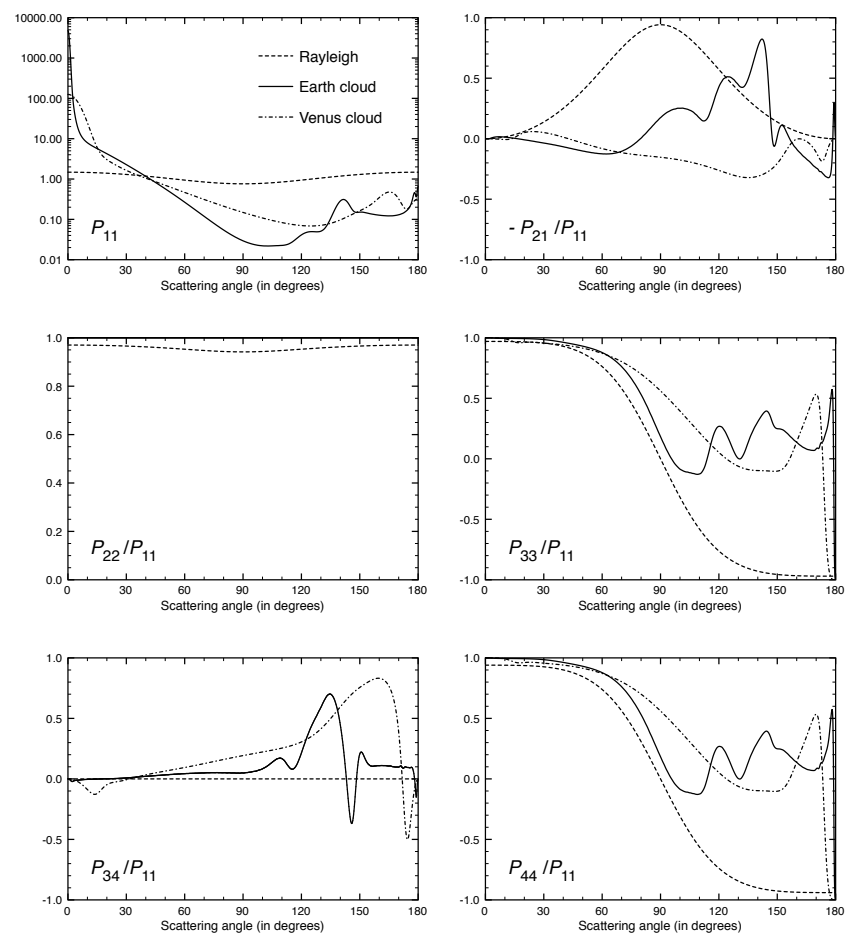

Fig. 2. Single scattering matrix elements of Earth-like cloud particles (solid lines), Venus-like cloud particles (dashed-dotted lines), and Rayleigh scattering molecules (dashed lines) at $0.55 \mu \mathrm{m}$. Elements $P_{11}$ have been normalized such that their averages over all scattering angles equal one, and the other elements have been normalized to $P_{11}$. For spherical particles, such as the cloud particles, $P_{22} / P_{11}=1$, $P_{34}=-P_{43}$, and $P_{33}=P_{44}$. The Earth-like cloud particles are described by a two-parameter gamma size distribution with $r_{\text {eff }}=8.0 \mu \mathrm{m}$ and $v_{\text {eff }}=0.1$. Their refractive index is $1.33+10^{-8} i$ (Hale \& Querry 1973). The Venus-like cloud particles have $r_{\text {eff }}=1.05 \mu \mathrm{m}, v_{\text {eff }}=0.07$, and a refractive index of $1.44+0.015 i$ (Hansen \& Hovenier 1974).

pixels have the same optical thickness, altitude, and particle properties.

The patchy clouds are described by $f_{\mathrm{c}}$, the fraction of all pixels on the whole disk that are cloudy, and by the actual distribution of the cloudy pixels across the disk. The patches are generated with the method described by Rossi \& Stam (2017), where cloudy pixels are defined by a 2D Gaussian, randomly placed on the square grid and which shape and orientation are chosen to simulate streaky, zonally oriented, clouds. Figure 5 shows examples of cloud patterns for $f_{\mathrm{c}}=0.3$ (i.e. $30 \%$ cloud cover) and $f_{\mathrm{c}}=0.5$.

For each cloud cover pattern across a planet, we define the asymmetry factor $\gamma$, which indicates the percentage of pixels (cloudy and non-cloudy) on one of the illuminated and visible hemispheres that do not have a similar pixel in a mirrored position on the other hemisphere (assuming that the planetary scattering plane divides the two hemispheres). If $\gamma=0.0$, all pixels have a mirror pixel (the circular polarization signal of this planet will equal zero), while if $\gamma=1.0$, none of the pixels has a mirror pixel. The asymmetry factors for the examples shown in Fig. 5 are 0.24 and 0.52 for the two $f_{\mathrm{c}}=0.3$ cases, and 0.28 and 0.61 for the two $f_{\mathrm{c}}=0.5$ cases.

\section{Results}

In this section, we will first show and discuss the reflected total and polarized fluxes for horizontally homogeneous planets 


\section{Earth-like cloud particles}
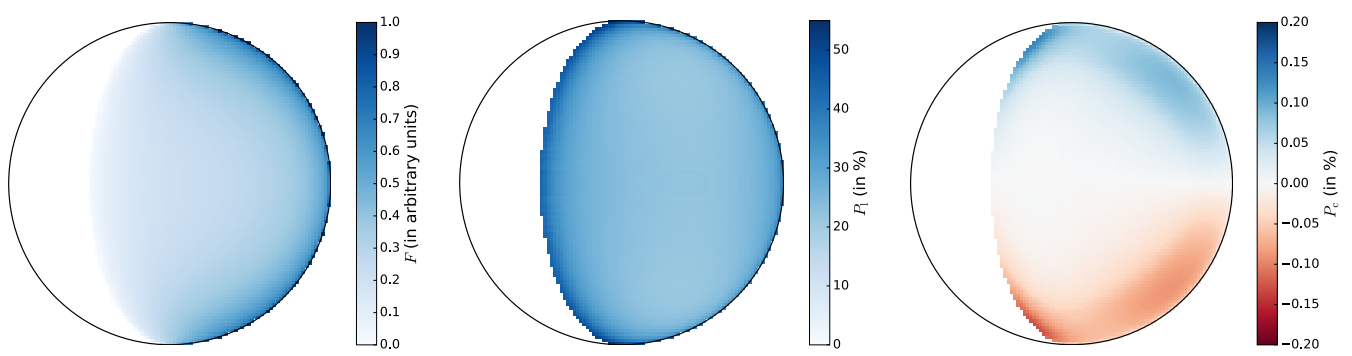

\section{Venus-like cloud particles}
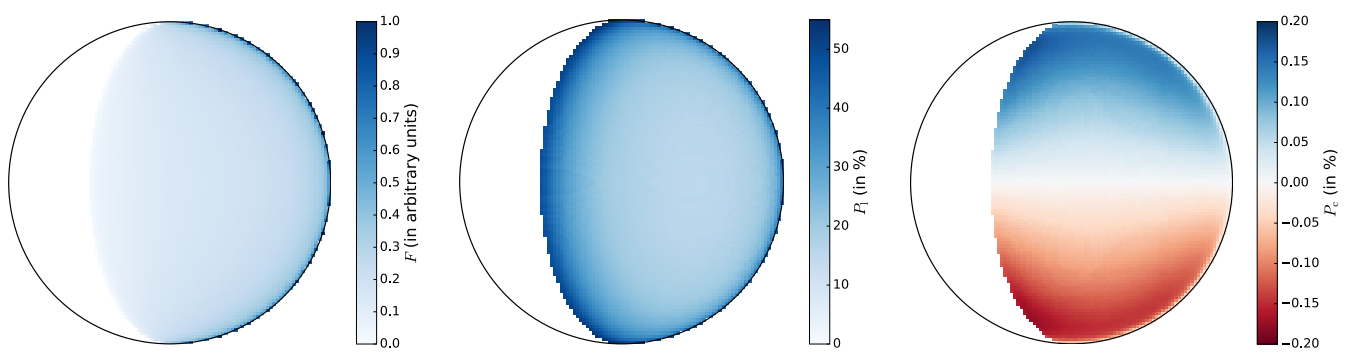

Fig. 3. Reflected light signals of planets with horizontally homogeneous clouds composed of Earth-like cloud particles (top row) or Venus-like cloud particles (bottom row), at a phase angle $\alpha$ of $60^{\circ}$. For each model planet, the cloud optical thickness $b_{\mathrm{c}}$ is 2.0 , the cloud bottom is at $2.0 \mathrm{~km}$, its top at $4.0 \mathrm{~km}$ (see Fig. 1), and the surface is black. Left column: total flux $F$ (normalized to the maximum on the disk); middle column: degree of linear polarization $P_{1}$; right column: degree of circular polarization $P_{\mathrm{c}}$. Integrated across the planetary disk, $P_{1}=25.5 \%$ for the Earth-like clouds and $23.8 \%$ for the Venus-like clouds, in both cases with the polarization direction perpendicular to the planetary scattering plane.

(Sect. 3.1), and then we will investigate the effects of horizontal inhomogeneities due to fractional cloud coverage (Sect. 3.2). For horizontally homogeneous planets, $P_{\mathrm{c}}$ will be zero when integrated across the planetary disk. For these planets, we will therefore only show and discuss signals of spatially resolved planets. Horizontally inhomogeneous planets can have a nonzero disk-integrated value of $P_{\mathrm{c}}$. The spatially resolved signals of these planets can straightforwardly be derived from the spatially resolved signals of the horizontally homogeneous planets. For the horizontally inhomogeneous planets, we will thus only discuss the spatially unresolved signals, which would be representative for exoplanet observations.

\subsection{Horizontally homogeneous planets}

As mentioned earlier, a planet with a purely gaseous atmosphere and a depolarizing or linearly polarizing surface will not reflect any circularly polarized flux: circularly polarized flux will only appear when the atmosphere contains aerosol and/or cloud particles. Figure 3 shows the reflected flux $F$, and the degrees of linear and circular polarization, $P_{1}$ and $P_{\mathrm{c}}$, respectively, for a planet at a phase angle $\alpha=60^{\circ}$ (the single scattering angle $\Theta$ is thus $120^{\circ}$ across the planetary disk). The horizontally and vertically homogeneous cloud layer has an optical thickness $b_{\mathrm{c}}=2.0$ (at $550 \mathrm{~nm}$ ), its bottom at $2.0 \mathrm{~km}$, and its top at $4.0 \mathrm{~km}$. The clouds are composed of either the Earth-like or the Venus-like particles.

It can be seen that for both particle types, the reflected flux $F$ is fairly constant across the planetary disk, thanks to the homogeneous layer of clouds that hides the dark surface. At the limb, $F$ (per pixel) is largest, because there the reflected light has been mostly scattered by the gas above the cloud, and the scattering phase function of gas molecules is relatively large at this phase angle (i.e. a scattering angle of $120^{\circ}$, see Fig. 2). At the terminator, the incident flux per atmosphere surface area is very small, yielding small values of $F$.

The degree of linear polarization, $P_{1}$, is also fairly constant across the disk, because it is strongly determined by the singly scattered light, and the single scattering angle is constant across the disk. Values of $P_{1}$ are somewhat higher at the limb and the terminator for both types of cloud particles because there the contribution of multiple scattered light from the lower layers, with usually a low degree of polarization, is relatively small, and there is a stronger contribution of relatively highly polarized light scattered by the molecules higher in the atmosphere. The smallest local values of $P_{1}$ for the Venus-like cloud particles are smaller than those for the Earth-like cloud particles. That is due to the difference in their single scattering polarization at this phase angle (cf. Fig. 2): indeed, were it not for the gaseous molecules overlying the cloud layer, the Venus-like cloud particles would yield a planet with a polarization direction parallel to the reference plane. Both the gas molecules and the Earthlike cloud particles yield perpendicular polarization. Integrated across the planetary disks, $P_{1}=25.5 \%$ for the planet with the Earth-like clouds and $24.5 \%$ for the planet with the Venus-like clouds at this phase angle.

The degree of circular polarization, $P_{\mathrm{c}}$, depends on the multiple scatterings involving a cloud particle at least once. As expected, it is generally small for both types of cloud particles, and the pattern of $P_{\mathrm{c}}$ on the northern hemisphere mirrors that on the southern hemisphere, except with the opposite sign, because of the horizontal homogeneity of the cloud cover. Along the equator, $P_{\mathrm{c}}$ equals zero due to symmetry. Because of this antisymmetry of the circular polarization on the planetary disk, $P_{\mathrm{c}}=$ $0 \%$ for both planets when integrated across the planetary disk.

Through the multiple scattering, $P_{\mathrm{c}}$ depends on the illumination and viewing geometries (hence on the phase angle and the location on the planet) and on the composition and structure of 
the planetary atmosphere and surface (the latter is black for our model planets). In particular, the gas molecules above the clouds appear to have a significant influence on $P_{\mathrm{c}}$, because they scatter linearly polarized light onto the clouds, and the cloud particles can subsequently scatter light back to space in a circularly polarized state, through the single scattering matrix element $P_{43}$ (see Fig. 2). Because this element has the same sign for the Earthlike and the Venus-like cloud particles, $P_{\mathrm{c}}$ across the planet has the same sign for both cloud types. The higher value of element $P_{43}$ of the Venus-like particles is responsible for the higher values of $P_{\mathrm{c}}$ across the planet with the Venus-like clouds. Another reason for the difference in the absolute value of $P_{\mathrm{c}}$ is the absorption within the Venus-like cloud particles (their single scattering albedo is 0.75 versus almost 1.0 for the Earth-like particles), which suppresses the multiple scattering of light between cloud particles. Because multiple scatterings between cloud particles randomize and hence usually lower the degree of polarization, less multiple scatterings will usually yield a higher $P_{\mathrm{c}}$.

The relative importance of the scattering by the gas and the cloud layer on $P_{\mathrm{c}}$ can be analysed more in depth by unravelling the different contributions. Figure B.1 shows $P_{\mathrm{c}}$ for five model atmospheres with varying optical thicknesses of the gas and the cloud, for the two cloud types. The vertical extension of the cloud is from 2.0 to $4.0 \mathrm{~km}$, like in Fig. 3. When filled with gas (models A, B, and C), the optical thickness of the lowest layer is 0.020 , otherwise (models D and E) it is zero. The gaseous optical thickness of the second layer is zero (models C and D) or 0.016 (models A, B, and E), and that of the third layer is zero (models B-D) or (cf. Fig. 1) $0.024+0.025+0.011=0.060($ models $\mathrm{A}$ and $\mathrm{E}$ ). By comparing the signals for the different model atmospheres, it is clear that the gas above the cloud strongly influences $P_{\mathrm{c}}$ : the linearly polarized light scattered downwards by the gas yields circularly polarized light through the single scattering matrix element $P_{34}$ (which equals $-P_{43}$ ) of the cloud particles for models $\mathrm{A}$ and E. Because element $P_{34}$ has the same sign across most of the single scattering angle range for the two cloud particle types (see Fig. 2) and because the incident linearly polarization field of the single scattered light is the same, the sign of $P_{\mathrm{c}}$ is the same for the two types of planets.

For the atmospheres without gas above the cloud (models B-D), $P_{\mathrm{c}}$ shows a pattern that depends on the cloud particle type, as it is mostly due to light that has been scattered twice by the cloud particles. The difference in the $P_{\mathrm{c}}$ pattern is to be expected because the direction of linear polarization of the singly scattered light is different for the different cloud particle types across most of the single scattering angle range, while the direction of circular polarization is the same across the scattering angle range for the different cloud particle types (cf. Fig. 2). In the absence of (a significant amount of) gas above the clouds, the pattern of $P_{\mathrm{c}}$ across the planet will also depend slightly on the gas within and/or below the clouds. This can be seen by comparing the plots for models B-D in Fig. B.1. This gas within and/or below the clouds can scatter linearly polarized light towards the cloud particles that can subsequently be scattered as circularly polarized light, and the light scattered by the gas can dilute $P_{\mathrm{c}}$ by adding unpolarized light to the signal emerging from the atmosphere.

Next, we investigate the effects of the vertical position of a cloud in a planetary atmosphere (with gas in all atmospheric layers). This position influences $P_{\mathrm{c}}$ because it influences the optical path lengths in the atmosphere, and hence, for example, the amount of linearly polarized light that is incident on the cloud from above and/or from below. Figure B.2 shows the spatially resolved $P_{\mathrm{c}}$ for the two cloud types with $b_{\mathrm{c}}=2.0$ with a cloud top altitude $z_{\mathrm{t}}$ equal to 2, 4, 8, 16, and $100 \mathrm{~km}$ (see Fig. 1, the cloud fills its atmospheric layer). A cloud top altitude of $100 \mathrm{~km}$, thus at the top of the atmosphere, is unrealistic, but has been included to gain insight in the light scattering processes.

Figure B.2 shows that increasing $z_{\mathrm{t}}$ up to at least $z_{\mathrm{t}}=8 \mathrm{~km}$, leads to a decrease of $P_{\mathrm{c}}$ for both cloud types. This decrease is due to the decrease of the linearly polarized, Rayleigh scattered flux that is incident on the top of the cloud. When $z_{\mathrm{t}}$ is increased even further, to 16 and $20 \mathrm{~km}$, the contribution of the gas below the cloud layer to $P_{\mathrm{c}}$ becomes significant. The difference in the amount of gas below the cloud is responsible for the difference in the patterns between the planet plots for model B in Fig. B.1 (a low cloud, hence little gas below the cloud) and those for $\alpha=60^{\circ}$ and $z_{\mathrm{t}}=100 \mathrm{~km}$ in Fig. B.2.

Not only the gas optical thickness influences the optical path through the atmosphere and hence $P_{\mathrm{c}}$, but also the cloud optical thickness $b_{\mathrm{c}}$. Figure B.3 shows $P_{\mathrm{c}}$ for Earth-like and Venuslike particles, and for $b_{\mathrm{c}}$ equal to $0.5,1.0,2.0,4.0$, and 10.0 at $\alpha$ equal to $30^{\circ}, 60^{\circ}, 90^{\circ}$, and $120^{\circ}$. The cloud top in all cases is at $4 \mathrm{~km}$. For the model atmospheres with Earth-like cloud particles, $P_{\mathrm{c}}$ seems largest for $b_{\mathrm{c}}=2.0$. For smaller $b_{\mathrm{c}}$, the amount of multiple scattering is too small to induce significant circularly polarized flux, while for increasing $b_{\mathrm{c}}$ above 2.0, the randomizing influence of the increasing multiple scattering decreases $V$ while increasing $F$, and thus decreases $P_{\mathrm{c}}$. The latter effect seems smallest at $\alpha=120^{\circ}$, because there the optical path lengths for the light reaching the observer are already very long and increasing $b_{\mathrm{c}}$ does not significantly change the contribution of multiple scattered light to the received signal.

The atmospheres with the Venus-like cloud particles show a different behaviour at least for $\alpha=30^{\circ}, 60^{\circ}$, and $90^{\circ}: P_{\mathrm{c}}$ increases with increasing $b_{\mathrm{c}}$ until $b_{\mathrm{c}}=2.0$, and then remains more or less constant. The reason is that the Venus-like cloud particles absorb light, and hence prevent a strong increase in multiple scattered light with increasing $b_{\mathrm{c}}$ and thus prevent a decrease of $P_{\mathrm{c}}$.

In the results presented so far, the model atmospheres were bounded below by black surfaces. The influence of a reflecting surface on $P_{\mathrm{c}}$ is shown in Fig. 4, where $P_{\mathrm{c}}$ across the planet has been plotted for surface albedo's $A_{\mathrm{s}}$ equal to 0.0 (the standard model) to 0.8 . The surface reflection is Lambertian, that is, isotropic and unpolarized. The atmosphere is the standard model atmosphere (see Fig. 3). The cloud has its top at $4.0 \mathrm{~km}$ and $b_{\mathrm{c}}=2.0$. As expected, increasing $A_{\mathrm{s}}$ adds unpolarized light to the bottom of the cloud layer and decreases $P_{\mathrm{c}}$. This decrease of $P_{\mathrm{c}}$ with increasing $A_{\mathrm{s}}$ decreases with increasing $b_{\mathrm{c}}$.

In case the surface below the atmosphere reflects linearly polarized light, it can influence the pattern of $P_{\mathrm{c}}$ across the planet, as this light can be scattered by the cloud particles and become circularly polarized, but the overall effect is expected to be small because in order to have a significant effect, there should be a horizontally homogeneously polarizing surface in combination with a high surface albedo. Even if these surface characteristics were met, a cloudy atmosphere would lead to a relatively low and diffuse surface illumination, limiting the absolute amount of linearly polarized flux that would reach the clouds, especially with larger values of $b_{\mathrm{c}}$. A circularly polarizing surface, for example covered by vegetation or other organic materials (e.g. Sparks et al. 2012b; Patty et al. 2017), could directly contribute to a planet's circularly polarized flux, thus without requiring an additional scattering by cloud particles. However, if the surface reflection were independent of the 


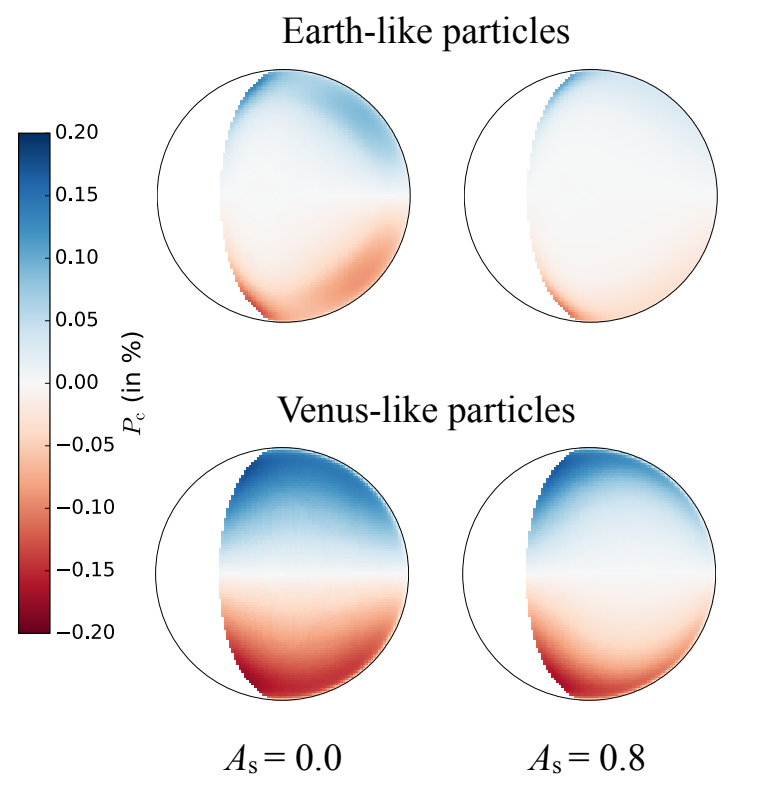

Fig. 4. Degree of circular polarization $P_{\mathrm{c}}$ for the standard model atmosphere with the cloud top at $4 \mathrm{~km}, b_{\mathrm{c}}$ equal to 2.0, and Earth-like (top) and Venus-like (bottom) cloud particles. The phase angle is $60^{\circ}$, and the surface albedo $A_{\mathrm{s}}$ equals 0.0 (left column) or 0.8 (right column).

azimuthal angle, for example, if the angular distribution of the reflected light were isotropic, the surface would leave no net circular polarization signal at the top of the atmosphere.

\subsection{Horizontally inhomogeneous planets}

We next investigate the influence of patchy cloud covers and the cloud cover variability on $P_{\mathrm{c}}$ of starlight that is reflected by a spatially unresolved planet, such as an exoplanet. As discussed above, the disk-integrated $P_{\mathrm{c}}$ of a horizontally homogeneous planet is zero, as contributions from one hemisphere cancel out those of the other hemisphere. Therefore, only horizontally inhomogeneous planets would yield non-zero values of $P_{\mathrm{c}}$. As Figs. B.2, B.3, and 4 show, $P_{\mathrm{c}}$ can vary significantly across a horizontally homogeneous cloudy planet, while it is zero in the absence of clouds. So patchy clouds could yield a net amount of circular polarization with $P_{\mathrm{c}}$ depending on the distribution of the patches across the planetary disk. Indeed, even for patchy clouds, any symmetry with respect to the scattering plane will cancel the net circular polarization. A strongly symmetric cloud cover across the planet would yield a relatively low net circular polarization signal, while the most extreme case, a planet with one fully cloudy and one cloud-free hemisphere (asymmetry parameter $\gamma$ would thus equal 1.0), could yield a relatively large net signal.

We use the cloud algorithm described in Sect. 2.3 (see Rossi \& Stam 2017; for a more detailed explanation) to generate 300 different patchy cloud covers for various values of the cloud coverage fraction $f_{\mathrm{c}}$ and compute the disk-integrated degree of circular polarization $P_{\mathrm{c}}$ for planets covered by those cloud patterns at phase angles ranging from $0^{\circ}$ to $180^{\circ}$. Examples of cloud patterns are given in Fig. 5.

Figure 6 shows as functions of $\alpha$, the maximum and minimum values of $P_{\mathrm{c}}$ as obtained with 300 different cloud patterns for cloud coverage fractions $f_{\mathrm{c}}$ ranging from 0.1 ( $10 \%$ clouds) to 0.9 (90\% clouds), for Earth-like and Venus-like cloud particles. The first thing to note are the overall very small values of $P_{\mathrm{c}}$ for these horizontally inhomogeneous planets: the maximum values
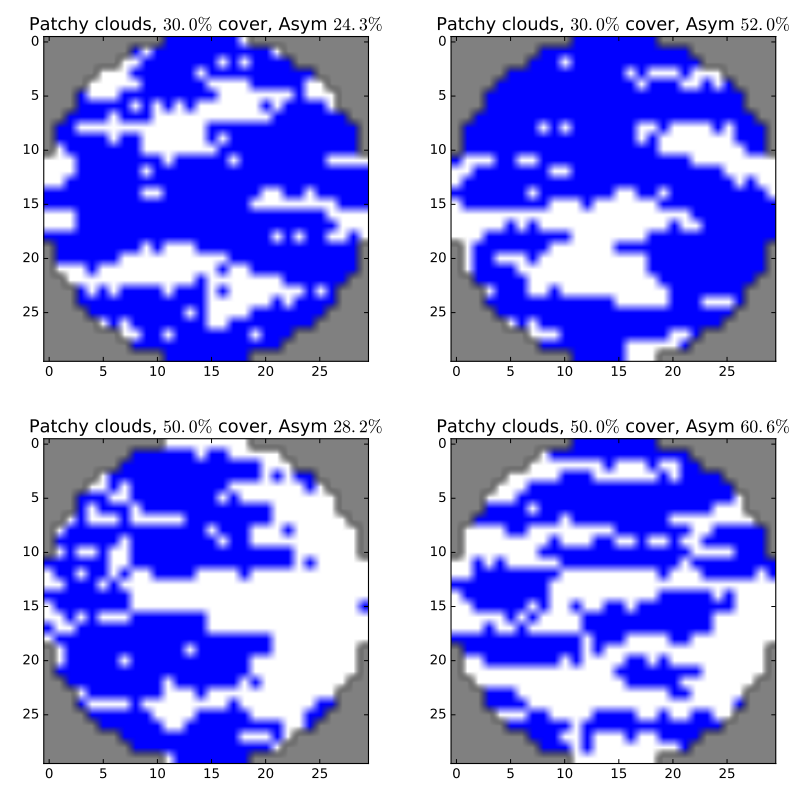

Fig. 5. Examples of patchy cloud patterns for $f_{\mathrm{c}}=0.3$ (top) and $f_{\mathrm{c}}=0.5$ (bottom) at $\alpha=0^{\circ}$. The asymmetry factors $\gamma$ of these planets are 0.24 (top left), 0.52 (top right), 0.28 (bottom left) and 0.61 (bottom right).

of $P_{\mathrm{c}}$ are about $0.020 \%$ for both the Earth-like, water clouds particles, and the Venus-like, sulfuric acid cloud particles. For both cloud types, these maximum values occur around $\alpha=50^{\circ}-60^{\circ}$. A second, smaller maximum occurs around $\alpha=130^{\circ}$, also for both cloud types. These (local) extreme values of $P_{\mathrm{c}}$ appear to be relatively independent of the cloud fraction $f_{\mathrm{c}}$; only for $f_{\mathrm{c}}=0.1$, the maximum values are slightly smaller.

Measuring $P_{\mathrm{c}}$ thus does not seem to provide insight into the cloud coverage fraction $f_{\mathrm{c}}$ of a planet. This might seem surprising, as on our model planets circularly polarized light is only induced by scattering by the cloud particles. However, it can be explained by looking at the occurrence of cloudy pixels on both sides of the planet's hemisphere, keeping in mind that the circularly polarized flux of a cloudy pixel on one hemisphere will be "cancelled" if the pixel's mirror pixel on the other hemisphere is also cloudy. At small cloud coverage fractions, there are few cloudy pixels to contribute circularly polarized flux, while the probability is small that a cloudy pixel has a cloudy mirror pixel on the opposite hemisphere that would cancel its polarized flux contribution. At large cloud coverage fractions, there are many cloudy pixels that contribute circularly polarized flux, but the probability that a cloudy pixel has a cloudy mirror pixel on the opposite hemisphere that cancels its polarized flux contribution is also large.

Apart from the minimum values of $P_{\mathrm{c}}$ at $\alpha=0^{\circ}$ and $180^{\circ}$, which are due to geometrical symmetry (note that $P_{\mathrm{c}}$ can deviate slightly from 0.0 at these phase angles due to the horizontal inhomogeneities), $P_{\mathrm{c}}$ shows a clear local minimum around $\alpha=100^{\circ}$, with a value of about $0.005 \%$ for the Earth-like cloud particles and $0.002 \%$ for the Venus-like cloud particles. These local minimum values of the disk-integrated $P_{\mathrm{c}}$ around $\alpha=100^{\circ}$ are related to the low spatially resolved values of $P_{\mathrm{c}}$ for $\alpha=90^{\circ}$ in Figs. B. 2 and B.3. Phase angles close to $90^{\circ}$ are usually considered to be the best for direct observations of exoplanets because there the angular distance between a planet and its star will be largest. Our results show that this phase angle range is, however, far from optimal for measurements of the circular polarization of exoplanets. The positive aspect of the low 

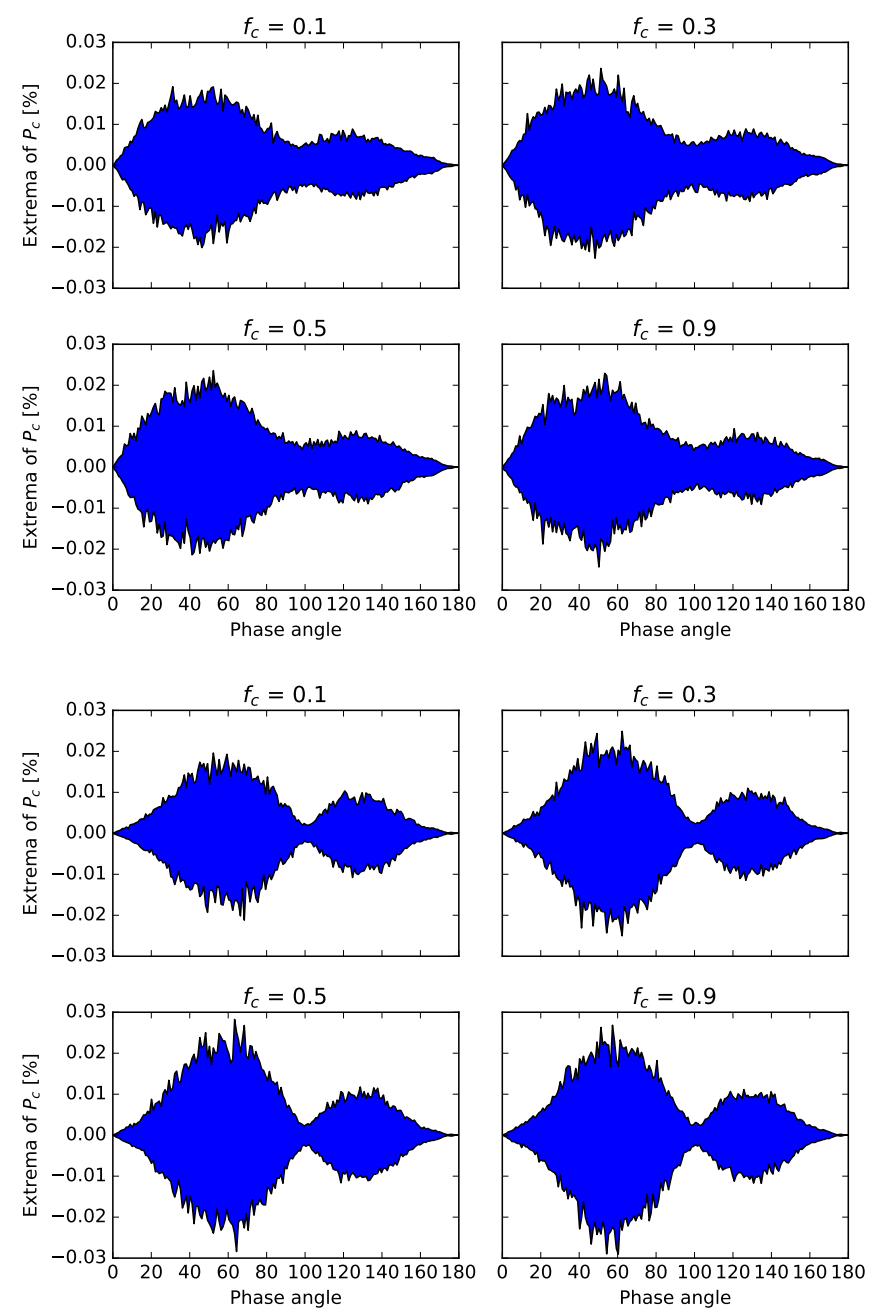

Fig. 6. Maximum values of $P_{\mathrm{c}}$ computed for 300 cloud patterns as a function of phase angle $\alpha$, for different cloud coverage fractions $f_{\mathrm{c}}$, and for Earth-like (top) and Venus-like cloud particles (bottom).

values of $P_{\mathrm{c}}$ in this phase angle range is that the influence of circularly polarized light on measurements of linear polarization, for example, through cross-talk between optical elements in an instrument or telescope, will be minimum in this phase angle range. Indeed, this phase angle range is in particular interesting for measurements of the state of the linear polarization of exoplanets with Rayleigh scattering atmospheres, for which $P_{1}$ will be large (Seager et al. 2000; Stam et al. 2004; Stam 2008).

The small differences in the general shape of the diskintegrated $P_{\mathrm{c}}$ as a function of $\alpha$ of the planets that are covered with water-clouds and those that are covered by sulfuric acid clouds, in particularly at small and large phase angles, are indicative of the different light scattering properties of the cloud particles (cf. Fig. 2), but will likely be far too subtle to be useful for cloud particle characterization. Indeed, the state of linear polarization, which has been proven to hold such information (see e.g. Hansen \& Hovenier 1974; Hansen \& Travis 1974), is expected to be much larger than $P_{\mathrm{c}}$ at most phase angles. The exception being the phase angles where $P_{1}=0$ (i.e. the so-called neutral points) but $P_{\mathrm{c}} \neq 0$.

Figure 7 shows the statistics of $P_{\mathrm{c}}$ computed for the 300 cloud patterns as functions of the cloud fraction $f_{\mathrm{c}}$ for $\alpha=50^{\circ}$ and $130^{\circ}$, the phase angles where the maximum values of $P_{\mathrm{c}}$ occur in Fig. 6. It can be seen that the distributions are quite symmetrical and have a median quite close to the average value (that is equal to $0.0 \%$ ), as it should be because the clouds can appear on both hemispheres. As can be seen, the range of values of $P_{\mathrm{c}}$ increases with increasing cloud coverage fraction $f_{\mathrm{c}}$ from 0.1 to $0.4-0.5$. Then it decreases with increasing $f_{\mathrm{c}}$, and it is particularly small for $f_{\mathrm{c}}=0.9$, for both phase angles. The very small range in $P_{\mathrm{c}}$ at the largest values of $f_{\mathrm{c}}$ is due to the planetary disk being almost completely cloudy and thus almost symmetric. At $\alpha=130^{\circ}$, only a narrow crescent of the planetary disk is illuminated and visible and the symmetry is even higher, and the range of $P_{\mathrm{c}}$ even smaller, than at $\alpha=50^{\circ}$. At $f_{\mathrm{c}}=0.1(10 \%)$, on the other hand, the disk is almost devoid of cloudy pixels and the range of $P_{\mathrm{c}}$ is thus small. Like in Fig. 6, the dependence of the range of $P_{\mathrm{c}}$ on $f_{\mathrm{c}}$ at intermediate cloud coverages is small.

\section{Discussion}

The results of our simulations show that the degree of circular polarization of planets with Earth- or Venus-like cloud particles is really small, especially compared to the linear polarization. We address here some limitations of this study and potential issues that observers trying to measure the circular polarization signals could face.

\subsection{Effects of non-Lambertian reflecting surfaces}

For a solid planet, the albedo $A_{\mathrm{s}}$ of the surface has a minor influence on the pattern of $P_{\mathrm{c}}$ across a cloudy planet. Increasing $A_{\mathrm{s}}$ generally decreases $P_{\mathrm{c}}$ as the surface reflects isotropically distributed and unpolarized light upwards. Even upon scattering by the cloud particles, this light will not add any net circularly polarized flux to the pattern of $P_{\mathrm{c}}$.

A linearly and/or circularly polarizing surface could produce a non-zero planetary $P_{\mathrm{c}}$, but only if the directional pattern of the linearly and/or circularly polarized flux is azimuthally anisotropic, if the polarizing surface covers a significant part of the planet, and if the diffuse sky flux that is incident on the surface is small compared to the incident direct sunlight or starlight. Due to the lack of azimuthally anisotropically reflecting polarized surface models, the model planets in this paper have Lambertian, that is depolarizing and isotropically reflecting, surfaces.

A thorough investigation of the actual strength of this effect requires a realistic surface reflection model, which is not available now. Circular polarization measurements have been proposed as a technique to search for chiral molecules that on Earth are related to the presence of life (Bailey 2000; Sparks et al. 2012b; Patty et al. 2017). Implementing a realistic surface reflection model, possibly based on circular polarization observations of light reflected by (regions on) the Earth or in a laboratory (Patty et al. 2017), thus appears to be a direction worthy of future research.

\subsection{Sources of noise}

Because we do not include background stellar flux in our computations of the degree of circular polarization, $P_{\mathrm{c}}$, the exoplanetary signals presented here apply to planets that are spatially resolved from their star. Our results can be adapted for background stellar flux at the location of the planet: if the stellar flux can be considered to be unpolarized it has to be added to the total planetary flux. It will thus lower the observable $P_{\mathrm{c}}$.

Circular polarization from the interstellar medium (ISM) or from the zodiacal light could also be a possible source of noise when trying to measure signals from an exoplanet. The zodiacal light is known to present circular polarization, as shown by 

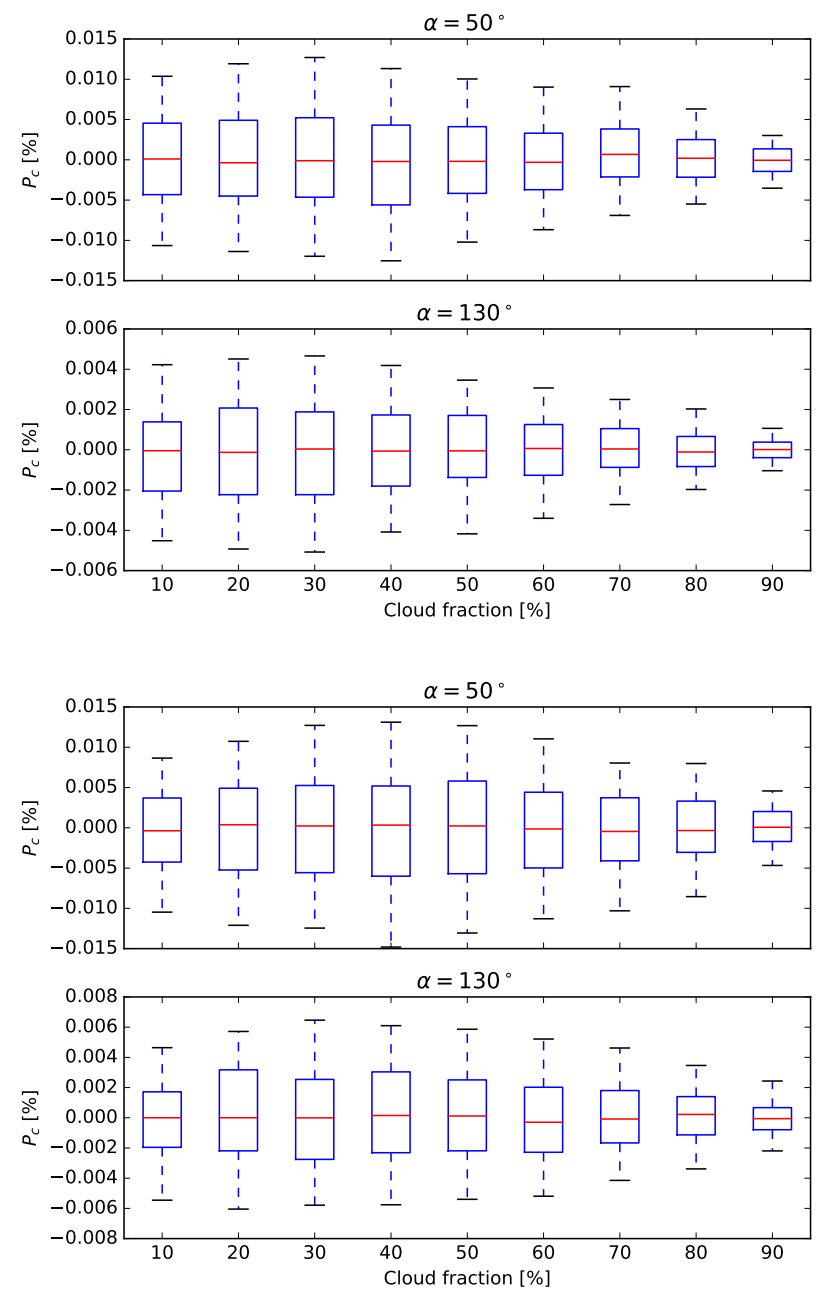

Fig. 7. Box-plots of the distribution of $P_{\mathrm{c}}$ over 300 cloud patterns, for $\alpha=50^{\circ}$ and $130^{\circ}$, and the Earth-like (top) and Venus-like (bottom) cloud particles. The box indicates the first and third quartiles, with the median in red. The whiskers show the fifth and 95th percentiles.

Wolstencroft \& Kemp (1972), with degrees of circular polarization up to $-0.8 \%$. We note that the visible-range circular polarization of the zodiacal light varies strongly with the solar longitude and that such measurements have not been reproduced since, leaving our understanding of the circular polarization of the zodiacal light incomplete. The ISM also produces polarized light, as non-spherical dust grains can be aligned along lines of magnetic fields, introducing dichroism and birefringence. The amount of circularly polarized light from the ISM shows values on the order of $10^{-4}$ (Kemp \& Wolstencroft 1972; Avery et al. 1975), which is close to our values for disk-integrated signals.

Nevertheless, the spectral dependence of the polarization due to dust in the visible is rather well known (Jones \& Whittet 2015). No significant changes are expected to occur on the timescale of observations of an exoplanet, especially when considering that the circular polarization of the planet will vary along the planetary orbit, which could help identify the planetary signal.

\subsection{Gaseous absorption}

This study ignores the effects of gaseous absorption. The degree of circular polarization in an absorption band could be different from that at continuum wavelengths, because more absorption implies less multiple scattering and a signal originating in higher atmospheric layers. These effects could yield less circular polarization, first because circular polarization arises from multiple scattering but also because higher layers are likely to be pure gas, which does not produce circular polarization. Less multiple scattering would also yield less unpolarized light. So the total degree of circular polarization in an absorption band could also increase with respect to that of the continuum. The change of $P_{\mathrm{c}}$ across an absorption band would thus critically depend on the amount of absorption and on the structure and composition of the atmosphere, just like the change of the degree of linear polarization across absorption bands, as can be seen in, for example, Fauchez et al. (2017) and Stam et al. (1999). A careful investigation of the behaviour of $P_{\mathrm{c}}$ in the presence of gaseous absorption requires a dedicated study when circular polarization observations in spectral regions with absorption bands are being planned, and is outside the scope of this paper.

\section{Summary}

We have computed the degree and direction of the circular polarization of planets, both spatially resolved and disk-integrated. The former would be applicable to solar system planets and the latter to exoplanets. Because sunlight or starlight that is incident on a planet is not circularly polarized, purely gaseous atmospheres do not yield any circularly polarized reflected light (cf. the single scattering matrix of Rayleigh scattering in Hansen \& Travis 1974), so all of our planets have cloudy atmospheres. Multiple scattering of incident unpolarized sunlight or starlight by gaseous molecules and then by cloud particles, or between cloud particles, will usually produce a circularly polarized signal.

Spatially resolved simulations of our horizontally homogeneous, cloudy model planets show that $P_{\mathrm{c}}$ is about a factor of 100 smaller than the degree of linear polarization $P_{1}$. Furthermore, $P_{\mathrm{c}}$ is mirror symmetric with respect to a planet's light equator (which coincides with the equatorial plane on our planets), except with the opposite sign. On the light equator, $P_{\mathrm{c}}$ is zero.

The pattern of $P_{\mathrm{c}}$ across the planetary disk depends on the type of cloud particles, and on the gaseous scattering optical thickness $b_{\mathrm{m}}$ above, within, and below the clouds, as gas scatters linearly polarized light onto the cloud particles that will subsequently be scattered as (partly) circularly polarized light. For both the water and the sulfuric acid particles comprising our model clouds, a significant amount of gas above the clouds yields positive (negative) $P_{\mathrm{c}}$ across the northern (southern) hemisphere at small phase angles, and negative (positive) $P_{\mathrm{c}}$ across the northern (southern) hemisphere at large phase angles. The sign change appears to happen at phase angles around $90^{\circ}$, except for very small cloud optical thicknesses $\left(b_{\mathrm{c}}=0.5\right.$ in Fig. B.3).

Increasing the cloud optical thickness $b_{c}$ increases the amount of light scattered between the cloud particles, and hence $P_{\mathrm{c}}$, for $b_{\mathrm{c}}$ up to about 2.0. Increasing $b_{\mathrm{c}}$ even further appears to decrease $P_{\mathrm{c}}$ slightly because then the multiple scattering appears to add mostly to the total flux $F$. With decreasing $b_{\mathrm{m}}$ above the clouds (i.e. with increasing cloud top altitude and/or increasing wavelength), the pattern of $P_{\mathrm{c}}$ due to the scattering of light between cloud particles becomes dominant. For our model clouds, this pattern shows sign changes and thus regions of zero $P_{\mathrm{c}}$ across each hemisphere.

For horizontally homogeneous planets, the disk-integrated value of $P_{\mathrm{c}}$ is zero, because the circular polarization on the southern hemisphere mirrors that on the northern hemisphere, except with opposite sign. For a horizontally inhomogeneous planet, such as a planet covered by patchy clouds, the 
disk-integrated $P_{\mathrm{c}}$ is usually not equal to zero. Knowing the values that can be expected for the disk-integrated $P_{\mathrm{c}}$ is important for exoplanetary detections. We therefore investigated the range of values that the disk-integrated $P_{\mathrm{c}}$ might attain for model planets covered by patchy clouds of two different compositions, with different cloud coverage percentages $f_{\mathrm{c}}$, and different spatial cloud patterns.

For various values of $f_{\mathrm{c}}$ and 300 different cloud patterns, the maximum values of the disk-integrated $P_{\mathrm{c}}$ are about $0.020 \%$ for both Earth-like and Venus-like cloud particles. These maximum values occur at phase angles $\alpha$ between about $50^{\circ}$ and $60^{\circ}$. A secondary, smaller maximum occurs around $130^{\circ}$. At $\alpha=0^{\circ}$ and $180^{\circ}$, the disk-integrated $P_{\mathrm{c}}$ equals zero due to symmetry (for the horizontally inhomogeneous planet, it can deviate slightly from zero). Another minimum of $P_{\mathrm{c}}$ is smaller than $0.005 \%$ and occurs around $\alpha=100^{\circ}$, both for the Earth-like and the Venuslike cloud particles. The relative independence of these values to $f_{\mathrm{c}}$, the cloud pattern, and the cloud particle type implies that measuring $P_{\mathrm{c}}$ of an exoplanet will provide little information on the cloud properties.

In the phase angle range where the disk-integrated $P_{\mathrm{c}}$ is relatively small, around $\alpha=100^{\circ}, P_{1}$, the degree of linear polarization, of a planet with an atmosphere in which gaseous molecules are the main scatterers, will actually be relatively large (Stam et al. 2004; Stam 2008). In this phase angle range, an exoplanet in a (more or less) circular orbit will have the largest angular distance from its star and thus be a good target for direct measurements of the exoplanet's flux and/or linear polarization signal. Our simulations show that when designing instruments for such measurements, the influence of the circular polarization signal, for example, through cross-talk in the optical components, can likely be ignored.

With the aim of this study being to investigate what signals can be observed and their physical origin, we do not explicitly address here the use of circular polarization for characterization of the atmosphere. Measuring the patterns in circular polarization could in principle provide information about the cloud particle properties (size, composition, shape) and atmospheric structure (cloud altitude, thickness). Further research is needed to investigate which information $P_{\mathrm{c}}$ would provide that could not be obtained from flux and in particular linear polarization measurements, as $P_{1}$ is usually much larger than $P_{\mathrm{c}}$, and which accuracy would be required for such $P_{\mathrm{c}}$ measurements in order to be able to derive such additional information.

Spatially resolved observations of the visible and infrared circular polarization of the Earth from space would help to validate the radiative transfer codes, our results for the cloudy regions on the planet, and to provide measurements that can be used to develop a realistic circularly polarizing surface model to improve the codes. Such measurements could also be done in a laboratory setting, provided a range of illumination and viewing angles (also including the azimuthal direction) is covered.

Acknowledgements. L.R. acknowledges funding through the PEPSci Programme of NWO, the Netherlands Organisation for Scientific Research.

\section{References}

Avery, R. W., Michalsky, J. J., Stokes, R. A., \& Ekstrom, P. A. 1975, AJ, 80, 1026 Bailey, J. 2000, in Bioastronomy 99, eds. G. Lemarchand, \& K. Meech, ASP Conf. Ser., 213

Berdyugina, S., Berdyugin, A., Fluri, D., \& Piirola, V. 2011, ApJ, 728, L6

Bonner, W. A. 1991, Orig. Life Evol. Biosph., 21, 59

Cellino, A., Gil-Hutton, R., \& Belskaya, I. N. 2015, Asteroids, eds. L. Kolokolova, J. Hough, \& A.-C. Levasseur-Regourd (Cambridge: Cambridge University Press), 360

de Haan, J. F., Bosma, P. B., \& Hovenier, J. W. 1987, A\&A, 183, 371

de Rooij, W. A., \& van der Stap, C. C. A. H. 1984, A\&A, 131, 237

Fauchez, T., Rossi, L., \& Stam, D. M. 2017, ApJ, 842, 41

Hale, G. M., \& Querry, M. R. 1973, Appl. Opt., 12, 555

Han, Q., Rossow, W. B., \& Lacis, A. 1994, J. Clim., 7, 465

Hansen, J. E., \& Hovenier, J. W. 1974, J. Atmos. Sci., 31, 1137

Hansen, J. E., \& Travis, L. D. 1974, Space Sci. Rev., 16, 527

Hovenier, J. W., \& van der Mee, C. V. M. 1983, A\&A, 128, 1

Hovenier, J. W., Van Der Mee, C., \& Domke, H., eds. 2004, Transfer of Polarized Light in Planetary Atmospheres: Basic Concepts and Practical Methods (The Netherlands: Springer), Astrophysics and Space Science Library, 318

Jones, T., \& Whittet, D. 2015, Interstellar Polarization (Cambridge: Cambridge University Press), 147

Karalidi, T., Stam, D. M., \& Hovenier, J. W. 2012, A\&A, 548, A90

Kawata, Y. 1978, Icarus, 33, 217

Kemp, J. C., \& Wolstencroft, R. D. 1971, Nature, 232, 165

Kemp, J. C., \& Wolstencroft, R. D. 1972, ApJ, 176, L115

Kemp, J. C., Henson, G. D., Steiner, C. T., \& Powell, E. R. 1987, Nature, 326 270

Kiselev, N., Rosenbush, V., Levasseur-Regourd, A.-C., \& Kolokolova, L. 2015, Comets, eds. L. Kolokolova, J. Hough, \& A.-C. Levasseur-Regourd (Cambridge: Cambridge University Press), 379

Kostogryz, N. M., Yakobchuk, T. M., \& Berdyugina, S. V. 2015, ApJ, 806, 97

Lucas, P. W., Hough, J. H., Bailey, J. A., et al. 2009, MNRAS, 393, 229

MacDermott, A. J., Barron, L. D., Brack, A., et al. 1996, Planet. Space Sci., 44, 1441

Martin, W. E., Hesse, E., Hough, J. H., \& Gledhill, T. M. 2016, J. Quant. Spectrosc Radiat. Transf., 170, 131

McLean, W., Stam, D. M., Bagnulo, S., et al. 2017, A\&A, 601, A142

Meierhenrich, U. J., Thiemann, W. H.-P., Barbier, B., et al. 2002, Orig. Life Evol. Biosph., 32, 181

Nagdimunov, L., Kolokolova, L., \& Sparks, W. 2013, Earth Planets Space, 65 1167

Patty, C. H. L., Visser, L. J. J., Ariese, F., et al. 2017, J. Quant. Spectrosc Radiat. Transf, 189, 303

Rossi, L., \& Stam, D. 2017, A\&A, 607, A57

Schmid, H. M., Joos, F., \& Tschan, D. 2006, A\&A, 452, 657

Seager, S., Whitney, B., \& Sasselov, D. 2000, ApJ, 540, 504

Sparks, W. B., Hough, J. H., \& Bergeron, L. E. 2005, Astrobiology, 5, 737

Sparks, W. B., Hough, J., Germer, T. A., et al. 2009, Proc. Natl. Acad. Sci., 106 7816

Sparks, W., Germer, T. A., MacKenty, J. W., \& Snik, F. 2012a, Appl. Opt., 51, 5495

Sparks, W., Hough, J. H., Germer, T. A., Robb, F., \& Kolokolova, L. 2012b, Planet. Space Sci., 72, 111

Stam, D. M. 2008, A\&A, 482, 989

Stam, D. M., De Haan, J. F., Hovenier, J. W., \& Stammes, P. 1999, J. Geophys. Res., 104, 16843

Stam, D. M., Hovenier, J. W., \& Waters, L. B. F. M. 2004, A\&A, 428, 663

Swedlund, J. B., Kemp, J. C., \& Wolstencroft, R. D. 1972, ApJ, 178, 257

Tomasko, M. G., \& Doose, L. R. 1984, Icarus, 58, 1

Wolstencroft, R. D. 1976, Icarus, 29, 235

Wolstencroft, R. D., \& Kemp, J. C. 1972, ApJ, 177, L137 


\section{Appendix A: Comparison with Kawata (1978)}

The spatially resolved signals computed in Sect. 3.1 can be compared with those in Figs. 11-13 of Kawata (1978). The comparison is limited since Kawata (1978) used no Earth-like cloud particles. The phase angle is, however, $53^{\circ}$, which is close to our value of $60^{\circ}$. Kawata's cloud particles have the same effective radius and variance as our Venus-like cloud particles, but since he used different wavelengths from us, the refractive index is different (i.e. 1.46 at $340 \mathrm{~nm}, 1.433$ at $700 \mathrm{~nm}$, and 1.43 at $1000 \mathrm{~nm}$ ). Kawata used three atmosphere models. Model 1 has one layer of mixed gas and cloud particles. The gas scattering optical thickness at $365 \mathrm{~nm}$ is 6 , and the cloud optical thickness 128. Model 2 consists of a lower layer with only cloud particles and an upper layer of gas. Model 3 has a lower layer with mixed cloud particles and gas, and an upper layer of gas.

Kawata compares the signals of these model atmospheres at $380 \mathrm{~nm}$ where the gas has a large influence, and at $700 \mathrm{~nm}$, where the gas has little influence. His results for his Model 1 at $700 \mathrm{~nm}$ (Fig. 11 in Kawata 1978) should be comparable to those of our atmosphere Models B (the gas optical thickness in our atmospheric layers is only 0.036 ) and D with Venus-like particles. Indeed, our results show similar characteristics to those of Kawata: in the absence of significant scattering by gas above the clouds, the neutral band crossing the disk from north to south is similar (although in our model, the band is shifted somewhat to the limb), as are the signs of $P_{\mathrm{c}}$. We also find the increase of $P_{\mathrm{c}}$ towards to limb. Our Model D shows an additional neutral band close to the terminator that is absent in the results of Kawata. This difference is probably due to the slight differences in atmosphere models, refractive index, depolarization factor for the Rayleigh scattering, and/or phase angle.

The results of Kawata in case the contribution of gas above the clouds is significant (his Fig. 12) are also similar to ours, except that in our model atmospheres, the gas optical thickness is much smaller: the presence of gas removes the north-south neutral band, leaving mostly positive $P_{\mathrm{c}}$ across the northern hemisphere, with the highest values towards the limb. We do

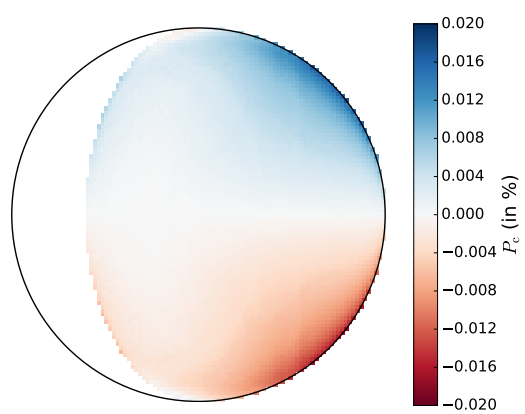

Fig. A.1. Degree of circular polarization $P_{\mathrm{c}}$ for $\alpha=53^{\circ}$ and a model atmosphere as close as possible to that used in Fig. 12 by Kawata: the atmosphere consists of a single layer with $b_{\mathrm{m}}=0.51$ and cloud particles with $b_{\mathrm{a}}=128$. The cloud particles are described by a two-parameter gamma size distribution with $r_{\text {eff }}=1.05 \mu \mathrm{m}$ and $v_{\text {eff }}=0.07$, with a refractive index equal to 1.458 .

not find the slightly negative region north of the equator and towards the terminator on the northern hemisphere (and reversed on the southern hemisphere) that shows up in Kawata's Fig. 12. Indeed, we adapted our model atmosphere, the cloud particles' properties, and the phase angle to simulate Kawata's Fig. 12 as closely as possible, and while then the overall shape of $P_{\mathrm{c}}$ across our planetary disk is very similar to that in Fig. 12 of Kawata, our maximum values (at the limb) are only about $0.017 \%$ while those found by Kawata appear to be higher than $0.020 \%$. We do find a slightly negatively polarized region on the northern hemisphere (and a slightly positively polarized region on the southern hemisphere) at approximately the same location as in Fig. 12 of Kawata, except with values that are a factor of ten smaller than those of Kawata. We also find small regions with inverse polarization near the poles that are absent in Fig. 12 of Kawata. The origin of these discrepancies is unknown, as not all details on Kawata's numerical computations are known to us, but they are extremely small, in particular in absolute sense, and leave us confident in our results. 
Appendix B: Additional figures

A.

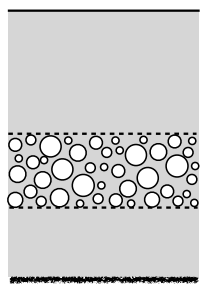

Earth-like particles
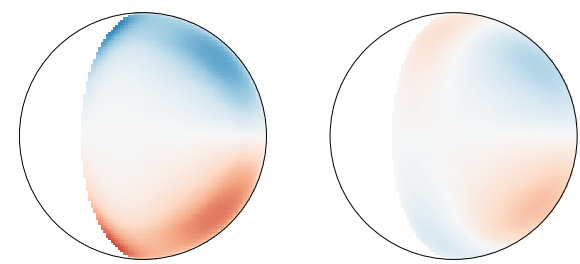

Venus-like particles

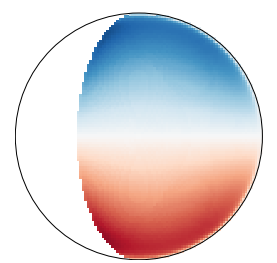

B.
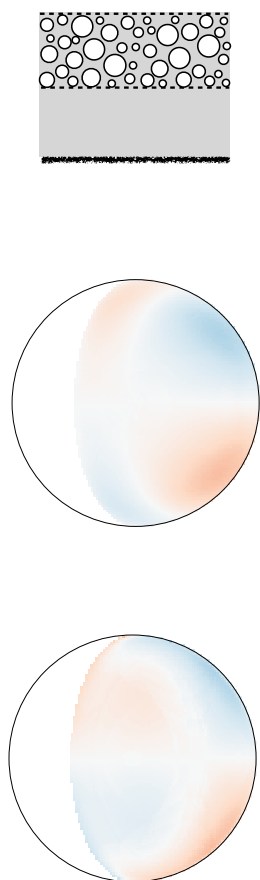

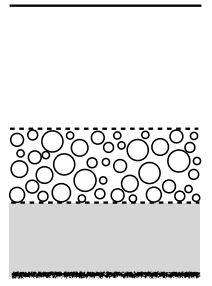

C.
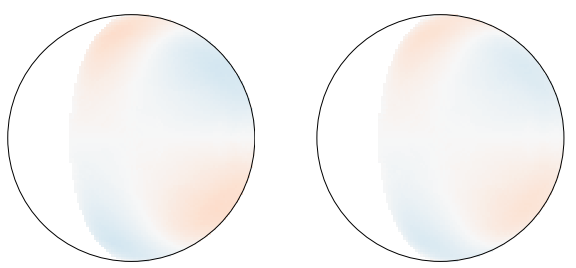

D.
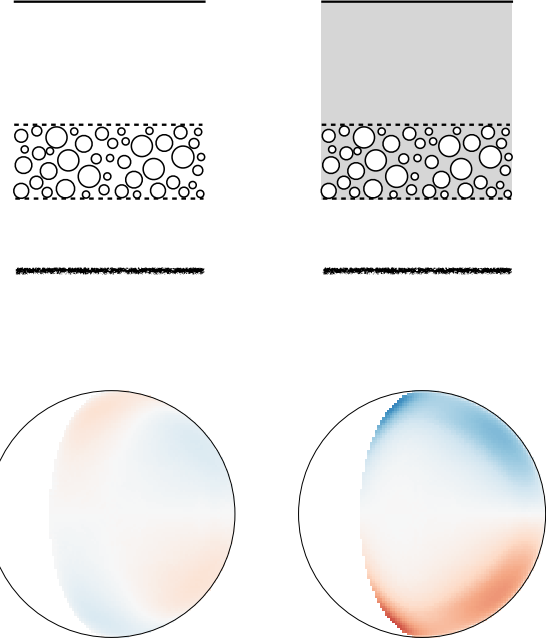

E.
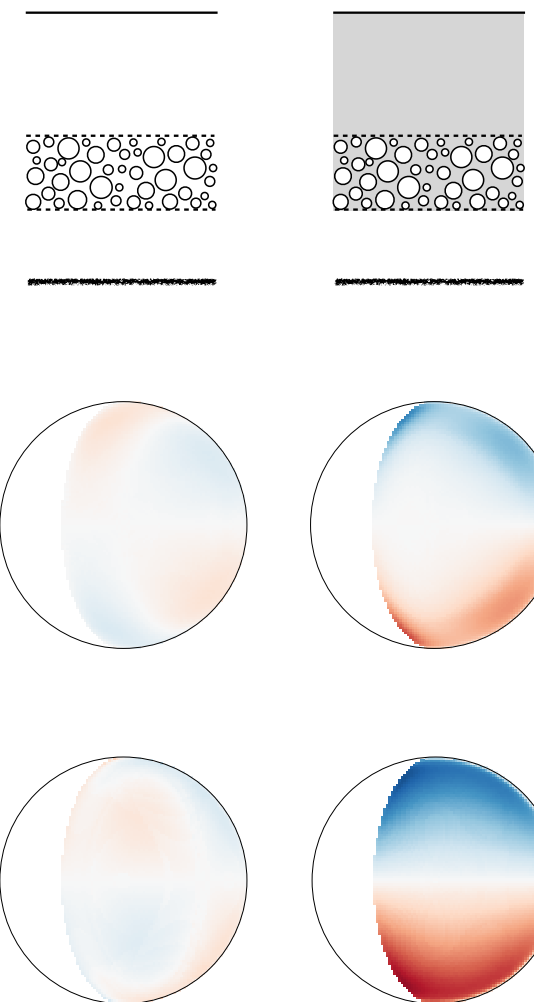

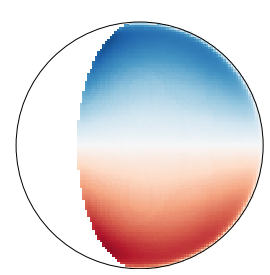

Fig. B.1. Similar to Fig. 3, except only for $P_{\mathrm{c}}$ and five different atmosphere models. The cloud top is at $4 \mathrm{~km}$, the subdivision of the atmosphere above the cloud as in Fig. 1 is not shown. From left to right: A. gas in all atmospheric layers (cf. Fig. 3); B. only gas in the bottom and in the cloud layer; C. only gas in the bottom layer; D. no gas at all; E. no gas in the bottom layer. 
L. Rossi and D. M. Stam: Circular polarization signals of cloudy (exo)planets

Earth-like particles
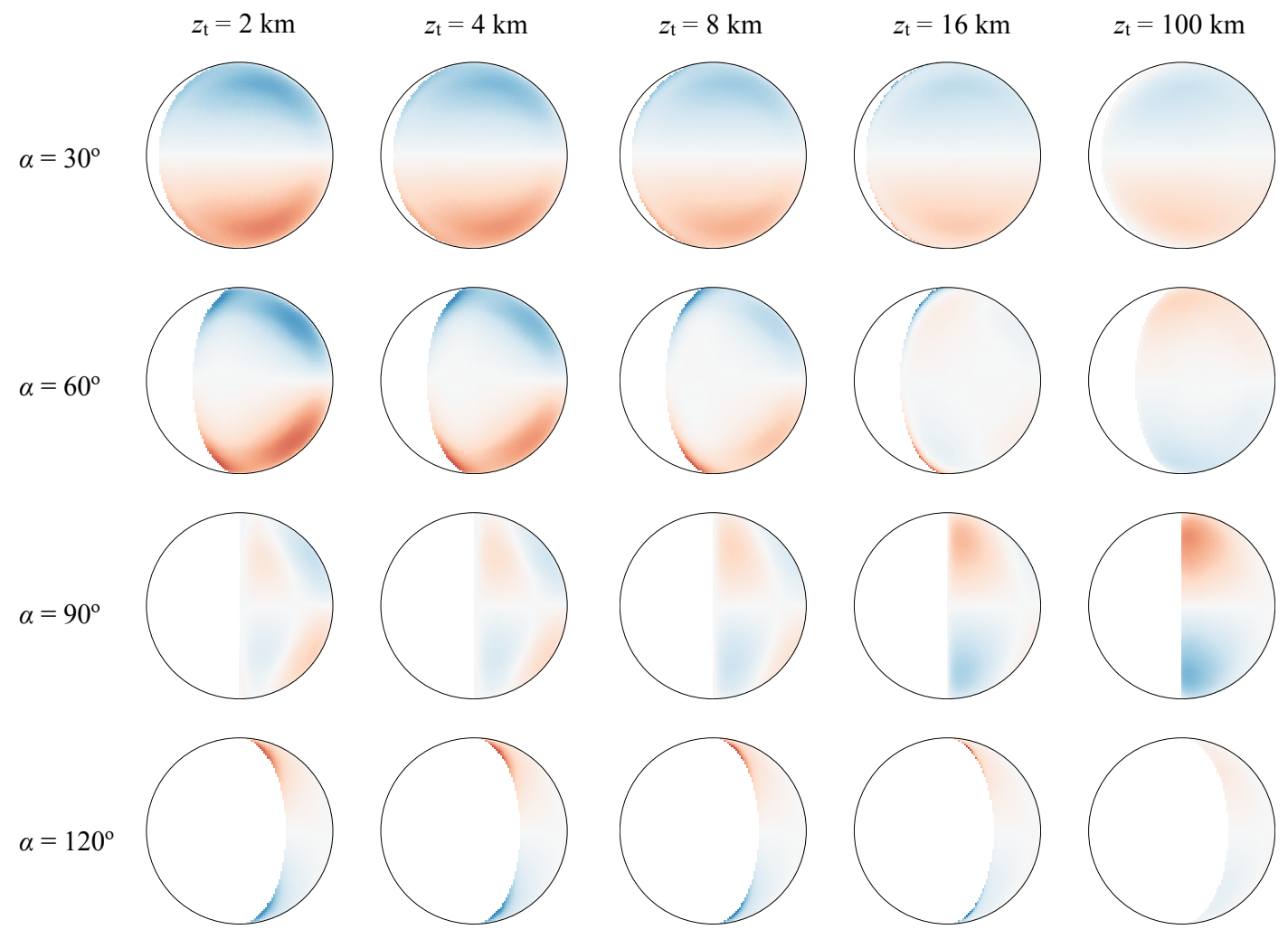

Venus-like particles
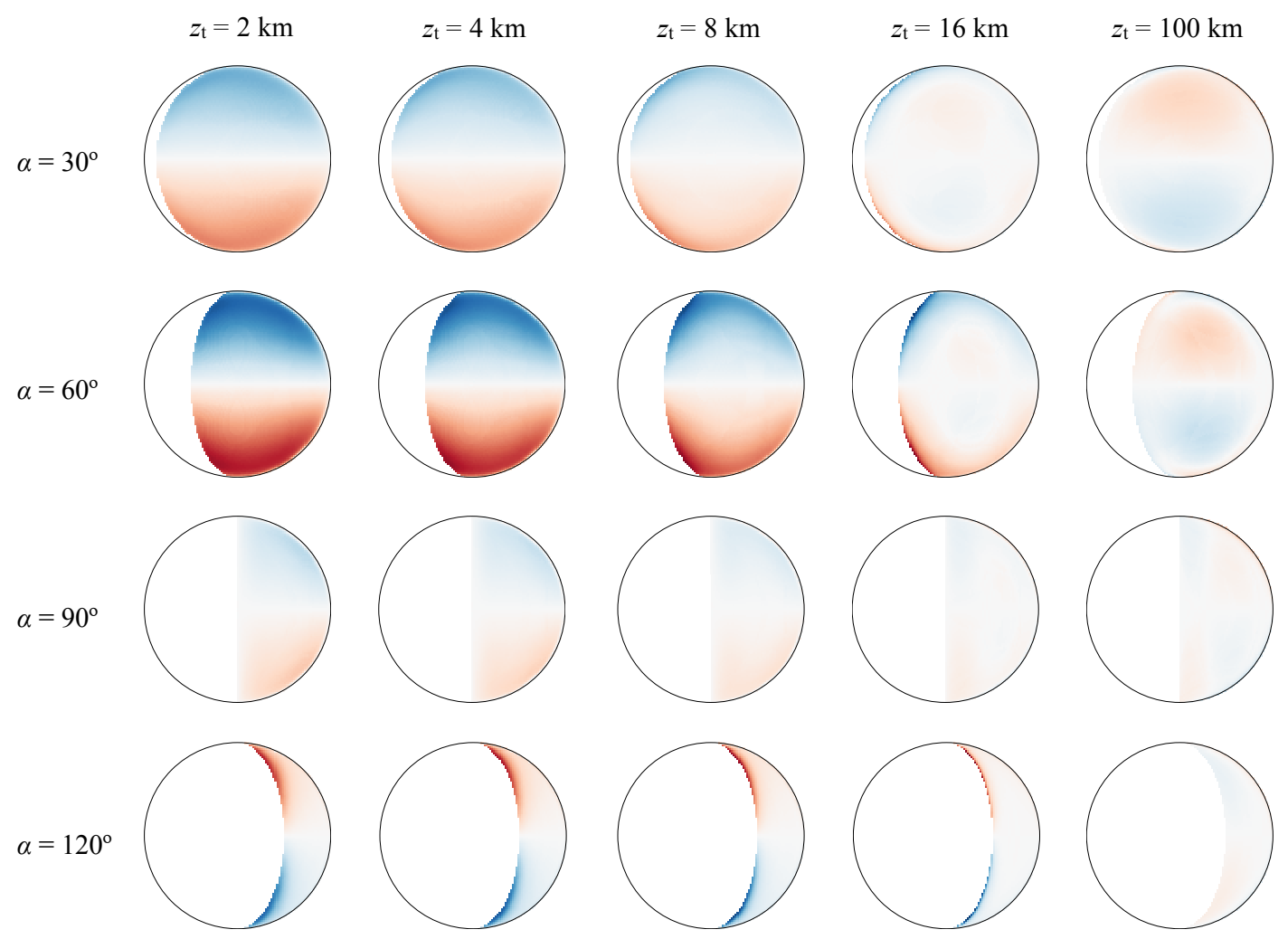

Fig. B.2. Degree of circular polarization $P_{\mathrm{c}}$ for $\alpha$ equal to $30^{\circ}, 60^{\circ}, 90^{\circ}$, and $120^{\circ}$. The standard model atmosphere has a $b_{\mathrm{c}}=2.0 \mathrm{cloud}$ consisting of Earth-like (top) or Venus-like (bottom) particles. The cloud top altitudes $z_{\mathrm{t}}$ are: $2 \mathrm{~km}$ (left columns), $4 \mathrm{~km}$ (cf. Fig. 3 ), 8 km, $16 \mathrm{~km}$, and $100 \mathrm{~km}$ (right columns). The colour scale is the same as that in Fig. 3. 
Earth-like particles

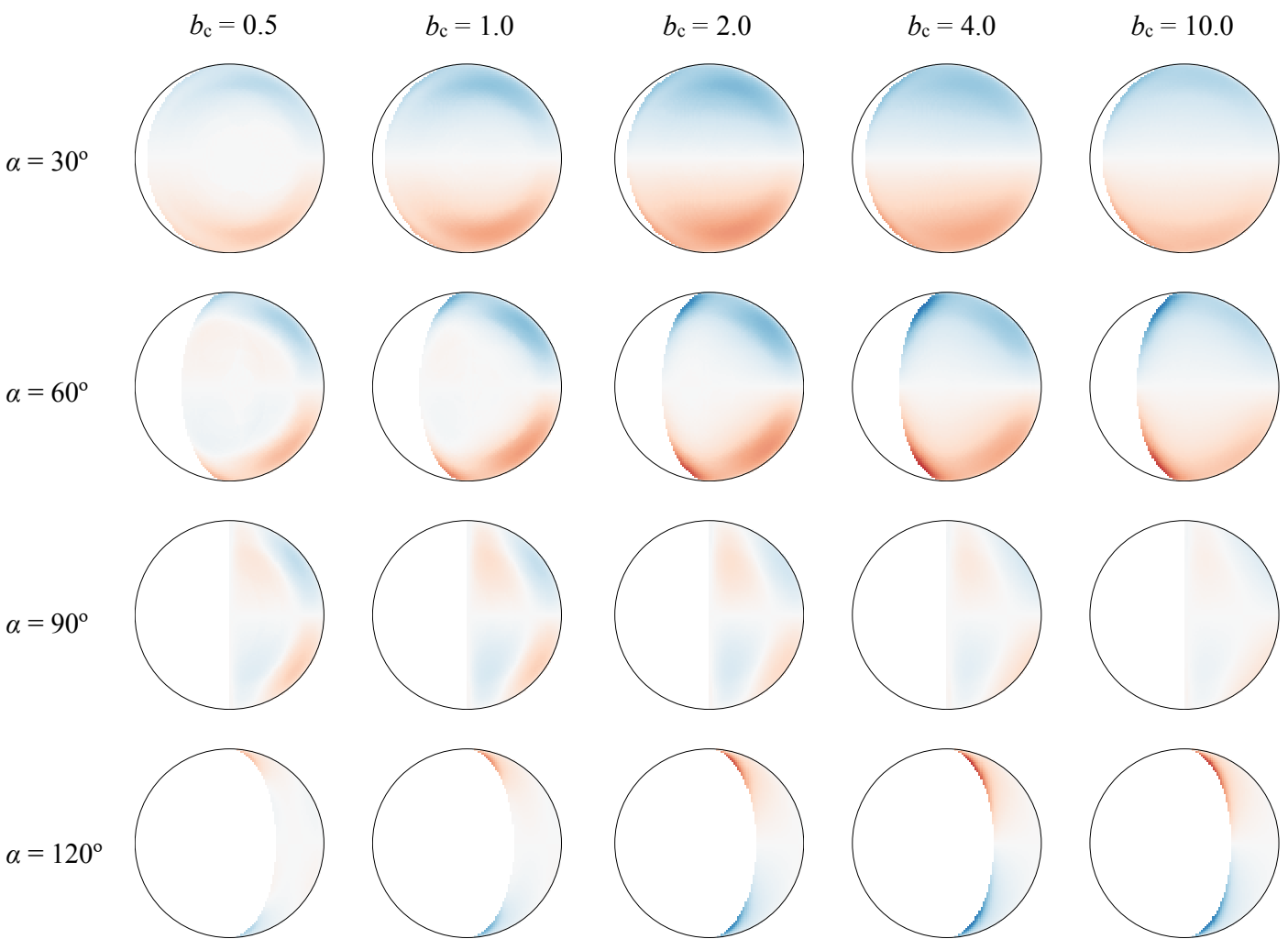

Venus-like particles

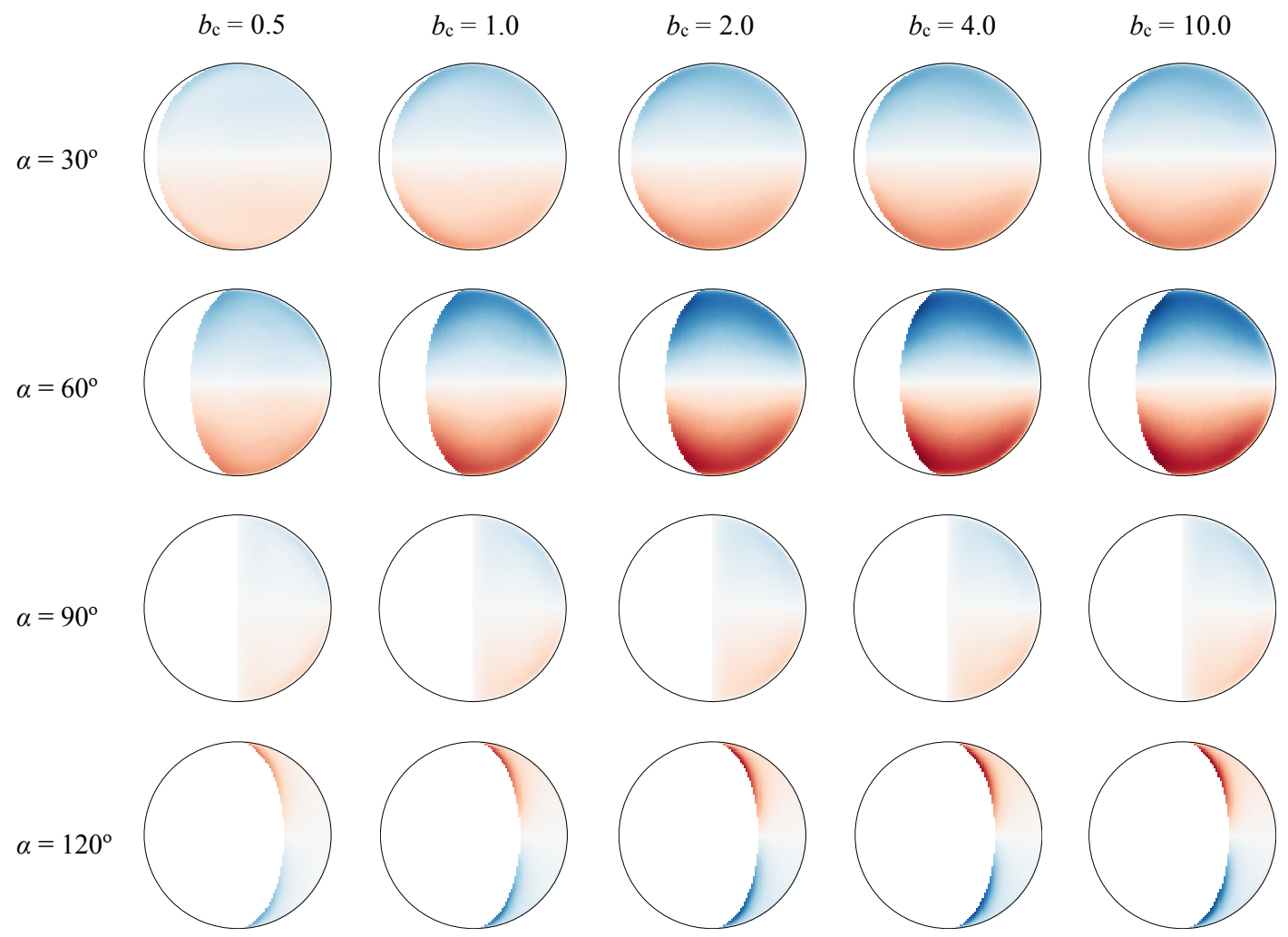

Fig. B.3. Degree of circular polarization $P_{\mathrm{c}}$ for $\alpha$ equal to $30^{\circ}, 60^{\circ}, 90^{\circ}$, and $120^{\circ}$. The standard model atmosphere has a cloud consisting of Earth-like (top) or Venus-like (bottom) particles, with the cloud top altitude $z_{\mathrm{t}}=4.0 \mathrm{~km}$, and the following cloud optical thicknesses $b_{\mathrm{c}}: 0.5$ (left columns), 1.0, 2.0, 4.0, and 10.0 (right columns). The colour scale is the same as that in Fig. 3. 